\title{
Superb Microvascular Imaging Ultrasound for Cervical Carotid Artery Stenosis for Prediction of the Development of Microembolic Signals on Transcranial Doppler during Carotid Exposure in Endarterectomy
}

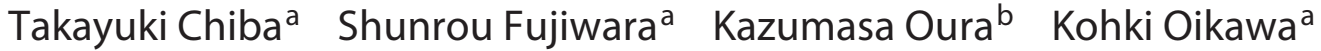 \\ Kokei Chida ${ }^{a}$ Masakazu Kobayashi ${ }^{a}$ Kenji Yoshida ${ }^{a}$ Yoshitaka Kubo $^{a}$

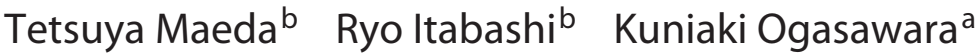 \\ ${ }^{a}$ Department of Neurosurgery, Iwate Medical University School of Medicine, Yahaba, Japan; ${ }^{b}$ Department of \\ Neurology and Gerontology, Iwate Medical University School of Medicine, Yahaba, Japan
}

\begin{abstract}
Keywords
Cervical carotid artery ultrasound · Superb microvascular imaging $\cdot$ Carotid endarterectomy $\cdot$ Microembolic signals
\end{abstract}

\begin{abstract}
Introduction: During exposure of the carotid arteries, embolism from the surgical site is recognized as a primary cause of neurological deficits or new cerebral ischemic lesions following carotid endarterectomy (CEA), and associations have been reported between histological neovascularization in the carotid plaque and both plaque vulnerability and the development of artery-to-artery embolism. Superb microvascular imaging (SMI) enables accurate visualization of neovessels in the carotid plaque without the use of intravenous contrast. This study aimed to determine whether preoperative SMI ultrasound for cervical carotid artery stenosis predicts the development of microembolic signals (MES) on transcranial Doppler (TCD) during exposure of the carotid arteries in CEA. Methods: Preoperative cervical carotid artery SMI ultrasound followed by CEA under TCD monitoring of MES in the ipsilateral middle cerebral artery was conducted
\end{abstract}

karger@karger.com www.karger.com/cee

Karger $\stackrel{\text { ' }}{5}$
(C) 2021 The Author(s)

Published by S. Karger AG, Basel

This is an Open Access article licensed under the Creative Common Attribution-NonCommercial-4.0 International License (CC BY-NC) (http://www.karger.com/Services/OpenAccessLicense), applicable to the online version of the article only. Usage and distribution for commercial purposes requires written permission. in 70 patients previously diagnosed with internal carotid artery stenosis (defined as $\geq 70 \%$ ). First, observers visually identified intraplaque microvascular flow (IMVF) signals as moving enhancements located near the surface of the carotid plaque within the plaque on SMI ultrasonograms. Next, regions of interest $(\mathrm{ROI})$ were manually placed at the identified IMVF signals (or at arbitrary places within the plaque when no IMVF signals were identified within the carotid plaque) and the carotid lumen, and time-intensity curves of the IMVF signal and lumen ROI were generated. Ten heartbeat cycles of both time-intensity curves were segmented into each heartbeat cycle based on gated electrocardiogram findings and averaged with respect to the IMVF signal and lumen ROI. The difference between the maximum and minimum intensities (ID) was calculated based on the averaged IMVF signal (ID $\left.\mathrm{IMVF}_{\mathrm{IMF}}\right)$ and lumen $\left(\mathrm{ID}_{\mathrm{I}}\right)$ curves. Finally, the ratio of ID IMVF to ID , was calculated. Results: MES during exposure of the carotid arteries were detected in 17 patients (24\%). The incidence of identification of IMVF signals was significantly greater in patients with MES (94\%) than in those without $(57 \% ; p=0.0067)$. The $I_{\text {IMVF }} / I_{\mid}$ratio was significantly greater in patients with MES $(0.108 \pm 0.120)$ than in those 
without $(0.017 \pm 0.042 ; p<0.0001)$. The specificity and positive predictive value for the $I D_{\mathrm{IMVF}} / \mathrm{ID}_{\text {| }}$ ratio for prediction of the development of MES were significantly higher than those for the identification of IMVF signals. Logistic regression analysis revealed that only the $\mathrm{ID}_{\mathrm{IMVF}} / \mathrm{ID}_{\text {| }}$ ratio was significantly associated with the development of MES $(95 \% \mathrm{Cl}$ 101.1-3,628.9; $p=0.0048$ ). Conclusion: Preoperative cervical carotid artery SMI ultrasound predicts the development of MES on TCD during exposure of the carotid arteries in CEA.

(C) 2021 The Author(s)

Published by S. Karger AG, Basel

\section{Introduction}

Carotid endarterectomy (CEA) has been shown to be capable of preventing stroke in appropriately selected patients [1-3]; however, 3 major cerebrovascular complications - cerebral embolism from the surgical site, hemispheric hypoperfusion during internal carotid artery (ICA) clamping, and cerebral hyperperfusion immediately after surgery - have been reported, and the rate of these complications can be reduced by appropriate perioperative measures taken by the surgeons [4]. Cerebral embolism from the surgical site is the principal cause of cerebrovascular complications from CEA [4], and such emboli can be detected as microembolic signals (MES) on intraoperative transcranial Doppler (TCD) monitoring of the middle cerebral artery (MCA) [4-6]. A significant correlation has been reported between detection of MES during exposure of the carotid arteries and new neurological deficits or ischemic lesions after CEA $[5,6]$. In the standard procedure for CEA, the carotid arteries are exposed in the order of the common, external, and ICA to secure space for clamping. When solid masses such as thrombi are present on the surface of ICA plaque, manipulation of the ICA for exposure may cause the masses to be dislodged and these masses run to the intracranial arteries in accordance with the pressure direction, leading to cerebral ischemic lesions [7]. Vulnerable plaque components are reported to be related to a histologically disrupted plaque surface, suggesting that thrombi are exposed to blood flow in carotid artery stenosis under such conditions [8]. Histological neovascularization has been shown to predict carotid plaque vulnerability $[9,10]$. In addition, local inflammatory damage and shear stress from the arterial lumen are known to lead to collapse, resulting in immature and fragile neovessels and intraplaque hemorrhage $[10,11]$. Thus, the degree of neovascularization in the carotid plaque may be associated with development of emboli from the surgical site during carotid artery exposure in endarterectomy [12].

Cervical carotid artery ultrasound has been widely used in the bedside evaluation of the morphology of this artery, especially for characterization of intraplaque components to identify vulnerable plaques, primarily hypoechoic plaques; however, a substantial overlap has been reported among the components of plaque echogenicity, even in quantitative evaluations such as those using a gray-scale median [13]. Furthermore, the development of MES during CEA cannot always be accurately predicted by plaque echogenicity $[12,14]$.

Superb microvascular imaging (SMI) is a new ultrasound imaging technique developed to overcome the limitations of conventional Doppler ultrasound by enabling visualization of neovessels without the need for intravenous contrast [15]. This technique applies an exclusive algorithm to differentiate true microvascular flow signals from wall motion artifacts and clutter, thereby allowing visualization of intraplaque microvascular flow (IMVF) signals [15]. Recent studies have demonstrated that SMI ultrasound can accurately detect neovascularization in the carotid plaque [15-18].

The purposes of the present study were to determine whether preoperative SMI ultrasound for cervical carotid artery stenosis can predict the development of MES on TCD during exposure of the carotid arteries in CEA and to propose practical clinical algorithms using SMI ultrasound and subsequent surgical management.

\section{Materials and Methods}

\section{Inclusion Criteria}

Patients with ipsilateral ICA stenosis (degree of stenosis $\geq 70 \%$, based on the North American Symptomatic Carotid Endarterectomy Trial [3]) on angiography with arterial catheterization and useful preoperative residual function (modified Rankin Scale score: 0-2) were included in this study. Patients who underwent CEA of the carotid bifurcation in our institution were finally included. Patients with chronic atrial fibrillation or a mechanical heart valve were excluded.

\section{Pre-, Intra-, and Postoperative Management}

All patients received dual antiplatelet therapy until the morning of the day of CEA. CEA was carried out by a senior neurosurgeon blinded to the preoperative ultrasound and intraoperative TCD findings under general anesthesia through a skin incision using an operative microscope. The senior neurosurgeon continued the procedure regardless of the intraoperative TCD findings. Heparin (5,000 IU) was administered as a bolus before clamping of the ICA.

Diffusion-weighted magnetic resonance imaging (DWI) was performed within 3 days before and $24 \mathrm{~h}$ after surgery. A neurora-
Chiba et al. 

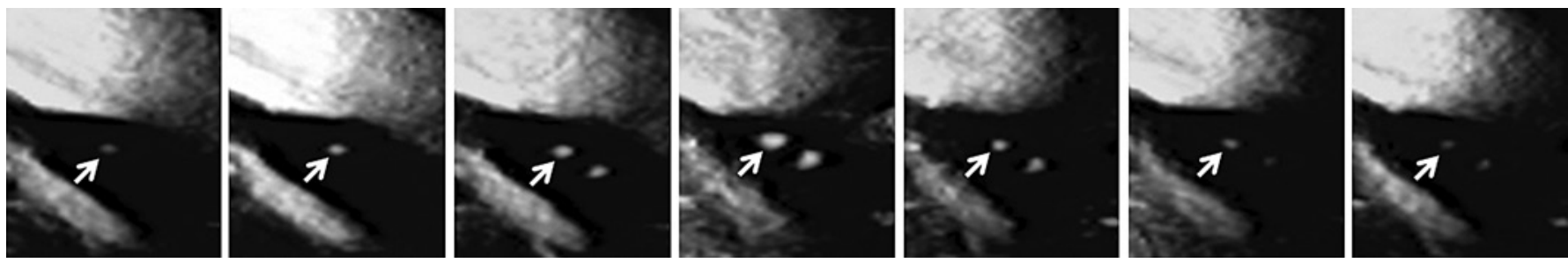

Fig. 1. SMI ultrasonograms of a 61-year-old man with symptomatic right ICA stenosis (95\%) showing MES during exposure of the carotid arteries in endarterectomy. The arrows indicate IMVF signals as moving enhancements located near the surface of the plaque. The intensity of the signal becomes stronger from the left-end panel to the middle panel and weaker from the middle panel to the right-end panel.

Fig. 2. a On the SMI ultrasonogram in Figure 1, each ROI is set at the IMVF signal within the carotid plaque $(\mathrm{P})$ and at the center within the lumen of the carotid artery proximal to the stenotic legion (L), respectively. b The black and red lines indicate the time-intensity curves of the IMVF signal and lumen ROIs, respectively. c The time-intensity curves of the IMVF signal and lumen ROI are segmented into 10 heartbeat cycles, and the durations of all 10 segmented time-intensity curves were made uniform. $\mathbf{d}$ These 10 segmented and uniformed time-intensity curves were averaged with respect to the IMVF signal and lumen ROI. The maximum and minimum intensities were determined (dotted lines) and the difference between the 2 intensities (maximum intensity - minimum intensity; ID) was calculated on the averaged IMVF signal (ID $\mathrm{IMVF}_{\mathrm{IMV}}$ ) and lumen $\left(\mathrm{ID}_{\mathrm{l}}\right)$ curves (vertical bidirectional arrows).
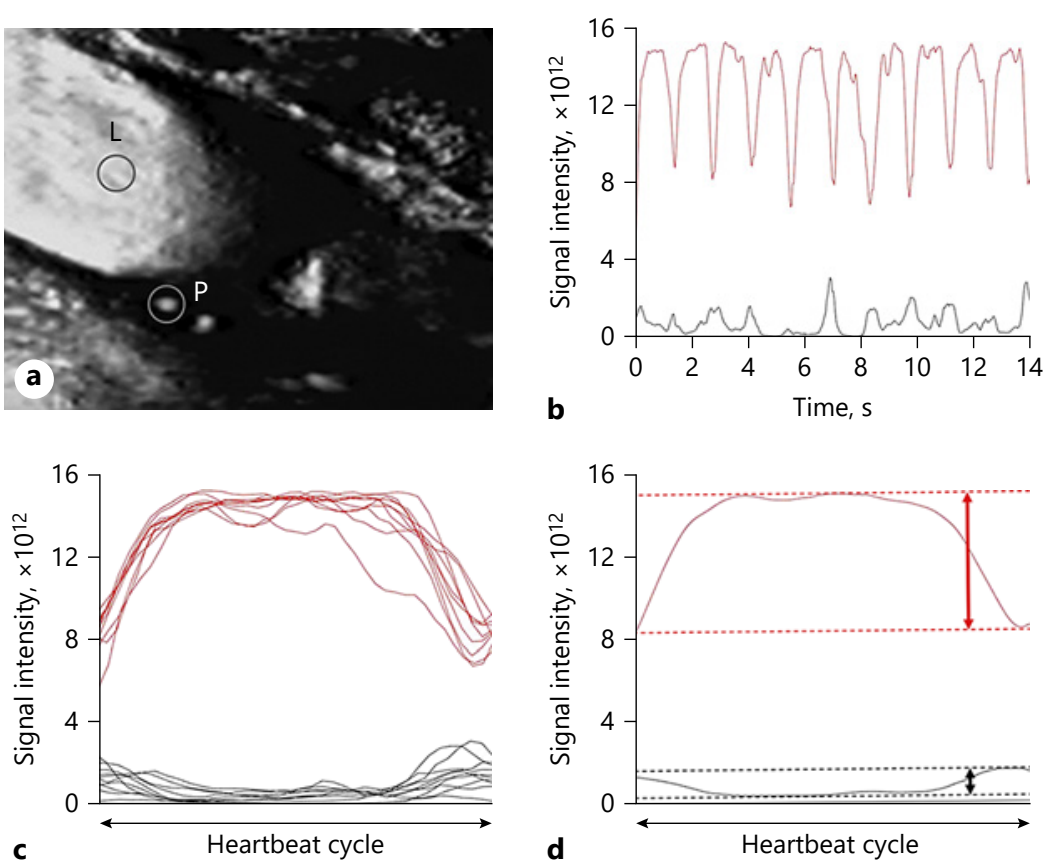

diologist who was blinded to the patients' clinical information was employed to analyze the DWI images and determine the development of new postoperative ischemic lesions.

\section{Cervical Carotid Artery Ultrasound and Data Processing}

Three days before the surgery, cervical carotid artery ultrasound (Canon Ultrasound System Aplio 700i; Canon Medical Systems, Otawara, Japan) with an $18-\mathrm{MHz}$ linear probe was performed on the affected side by a single investigator. All of the recorded images were stored on the scanner's hard disk drive. A carotid plaque was first identified on B, color Doppler, and pulse Doppler mode images. Next, SMI ultrasonography was performed using the monochrome SMI mode that showed a twin-view display of the plaque in B mode and monochrome SMI side by side with electrocardiogram gating [15]. The SMI-specific region of interest (ROI) box was positioned around the entire plaque under the following conditions: mechanical index of 1.0, frame rate of 28 frames/s, dynamic range of $65 \mathrm{~dB}$, and SMI velocity of $1.5 \mathrm{~cm} / \mathrm{s}$. Carotid plaques were observed on the longitudinal plane for $2 \mathrm{~min}$.

Two observers who were blinded to patient information visually excluded static enhancements and identified IMVF signals as moving enhancements located near the surface of the carotid plaque within the plaque on the SMI ultrasonograms (Fig. 1) [15]. Each investigator made independent assessments without information from the other to determine the interobserver variability of identification of the IMVF signal. When the results from the 2 observers were not in agreement, disagreements were resolved by consensus. When IMVF signals were identified within the carotid plaque, circular ROI were manually placed in the IMVF signals on the console so that they included the IMVF signals (Fig. 2a). When 2 or more IMVF signals were identified within the carotid plaque of one patient, ROI were placed in all these signals. When no IMVF signals were identified within the carotid plaque, 2 circular ROI were arbitrarily placed near the surface of the carotid plaque with- 
in the plaque so that these ROI did not include static enhancements. Regions with acoustic shadows due to calcification were also excluded from setting ROI. Next, the same investigator manually placed a circular ROI at the center within the lumen of the carotid artery proximal to the stenotic legion on the same image (Fig. 2a). These signal data from SMI ultrasonography were then transferred to the workstation. Time-intensity curves of the IMVF signal (arbitrary ROI signals were defined as IMVF signals when no IMVF signals were visually identified within the carotid plaque) and lumen ROI were generated using in-house software on MATLAB R2015b (MathWorks, Natick, MA; Fig. 2b), and 10 heartbeat cycles from both time-intensity curves were selected and segmented into each heartbeat cycle based on gated electrocardiogram findings. Because the duration of each heartbeat cycle differs slightly because of respiratory fluctuation and autonomic nerve function, even in healthy patients, the durations of all 10 segmented time-intensity curves were made uniform (Fig. 2c). These 10 uniform segmented time-intensity curves were then averaged with respect to the IMVF signal and lumen ROI (Fig. 2d). The maximum and minimum intensities were determined, and the difference between the 2 intensities (maximum intensity - minimum intensity; ID) was calculated based on the averaged IMVF signal $\left(\mathrm{ID}_{\mathrm{IMVF}}\right)$ and lumen $\left(\mathrm{ID}_{1}\right)$ curves (Fig. $\left.2 \mathrm{~d}\right)$. Finally, considering the influence of the carotid lumen signal on the IMVF signal, the ratio of $\mathrm{ID}_{\mathrm{IMVF}}$ to $\mathrm{ID}_{1}$ was calculated. When 2 or more ROI were placed within the carotid plaque in the same patient, the maximum $\mathrm{ID}_{\mathrm{IMVF}} / \mathrm{ID}_{1}$ ratio was selected for analysis.

\section{TCD Monitoring}

TCD was performed using the Pioneer TC2020 system (EME, Uberlingen, Germany; software version 2.50; $2-\mathrm{MHz}$ probe; diameter, $1.5 \mathrm{~cm}$; insonation depth, 40-66 mm; scale, -100 and +150 $\mathrm{cm} / \mathrm{s}$; sample volume, $2 \mathrm{~mm}$; 64-point fast Fourier transform; fast Fourier transform length, $2 \mathrm{~mm}$; fast Fourier transform overlap, $60 \%$; high-pass filter, $100 \mathrm{~Hz}$; detection threshold, $9 \mathrm{~dB}$; and minimum increase time, $10 \mathrm{~ms}$ ) for insonation of the MCA ipsilateral to the carotid artery undergoing CEA by a trained examiner with a headframe. TCD data were stored on a hard disk using a coding system and later analyzed manually by a clinical neurophysiologist blinded to the patient information. MES were identified during exposure of the carotid arteries (from skin incision to ICA clamping) according to the recommended guidelines [19].

\section{Statistical Analysis}

We determined the necessary sample size based on previous studies [12, 15]. Development of MES during exposure of the carotid arteries was estimated to occur in 10 and $50 \%$ of patients with a high $\mathrm{ID}_{\mathrm{IMVF}} / \mathrm{ID}_{1}$ ratio and a low $\mathrm{ID}_{\mathrm{IMVF}} / \mathrm{ID}_{1}$ ratio, respectively. We estimated that the ratio of the number of patients with a high $\mathrm{ID}_{\mathrm{IMVF}} / \mathrm{ID}_{1}$ ratio to that with a low $\mathrm{ID}_{\mathrm{IMVF}} / \mathrm{ID}_{1}$ ratio was 0.5 . Thus, 68 patients were required for a statistical power of $90 \%$ and an $a$ error of $5 \%$ to detect a $40 \%$ difference in the development of MES during exposure of the carotid arteries, with 2-sided significance. Considering patients who had no ROI within the carotid plaque because of an acoustic shadow due to heavy calcification in the whole plaque or reliable TCD monitoring due to failure to obtain an adequate bone window, we sought to enroll 90 patients into this study.

Data are expressed as means \pm SD. Weighted $\kappa$ statistics were calculated using data from the 2 observers to obtain the interob- server agreement of the number of IMVF signals. Relationships between the development of MES during exposure of the carotid arteries and each variable, including identification of the IMVF signal and $\mathrm{ID}_{\mathrm{IMVF}} / \mathrm{ID}_{1}$ ratio, were evaluated using the $\chi^{2}$ test or the Mann-Whitney U test. The relationships between new postoperative ischemic lesions on DWI and the $\mathrm{ID}_{\mathrm{IMVF}} / \mathrm{ID}_{1}$ ratio were evaluated using the Mann-Whitney U test. The accuracy of the ID $_{\text {IMVF }} /$ $\mathrm{ID}_{1}$ ratio to predict the development of MES during exposure of the carotid arteries was determined using a receiver operating characteristic (ROC) curve, and the ability to discriminate between the presence and absence of MES during exposure of the carotid arteries was estimated using the area under the ROC curve (AUC). The exact $95 \%$ CI for the sensitivity, specificity, and positive and negative predictive values were computed with a binomial distribution. Differences in the sensitivity, specificity, and positive and negative predictive values among identification of the IMVF signal and the $\mathrm{ID}_{\mathrm{IMVF}} / \mathrm{ID}_{1}$ ratio were analyzed using $95 \% \mathrm{CI}$. The sequential backward elimination approach was used for logistic regression analysis of variables related to the development of MES during exposure of the carotid arteries. Exclusion of factors was halted when the $p$ value of the remaining variables reached $<0.2$. For all statistical analyses, $p<0.05$ was considered statistically significant.

\section{Results}

A total of 90 patients met the inclusion criteria during the 22-month study period; among them, 4 who had visited our institute $<14$ days after the last ischemic attack and undergone urgent CEA because of crescendo transient ischemic attacks and did not subsequently undergo cervical carotid artery SMI ultrasound. The other 86 patients underwent cervical carotid artery SMI ultrasound prior to CEA, but in 3 of these patients no ROI could be placed within the carotid plaque, owing to an acoustic shadow caused by heavy calcification in the whole plaque; as a result, those 3 patients were excluded from the analysis. TCD was attempted in the remaining 83 patients, but due to the failure to obtain an adequate bone window 13 of those patients did not show reliable TCD monitoring throughout the entire operation and were therefore excluded from the analysis. Finally, 70 patients were enrolled into this study.

Among these 70 patients (63 men and 7 women, mean ( \pm SD) age $71 \pm 6$ years, range $49-82$ years), 61, 21, and 62 had hypertension, diabetes mellitus, and dyslipidemia, respectively. Fifty-seven patients reported ipsilateral carotid territory symptoms within the 6 months prior to surgery; 15 experienced onset of transient ischemic attacks alone, 22 had a minor stroke alone, and 20 had onset of a minor stroke after a transient ischemic attack. Seven patients with a lacunar/cardioembolic stroke underwent CEA more than 6 months after the last ischemic event and
Chiba et al. 


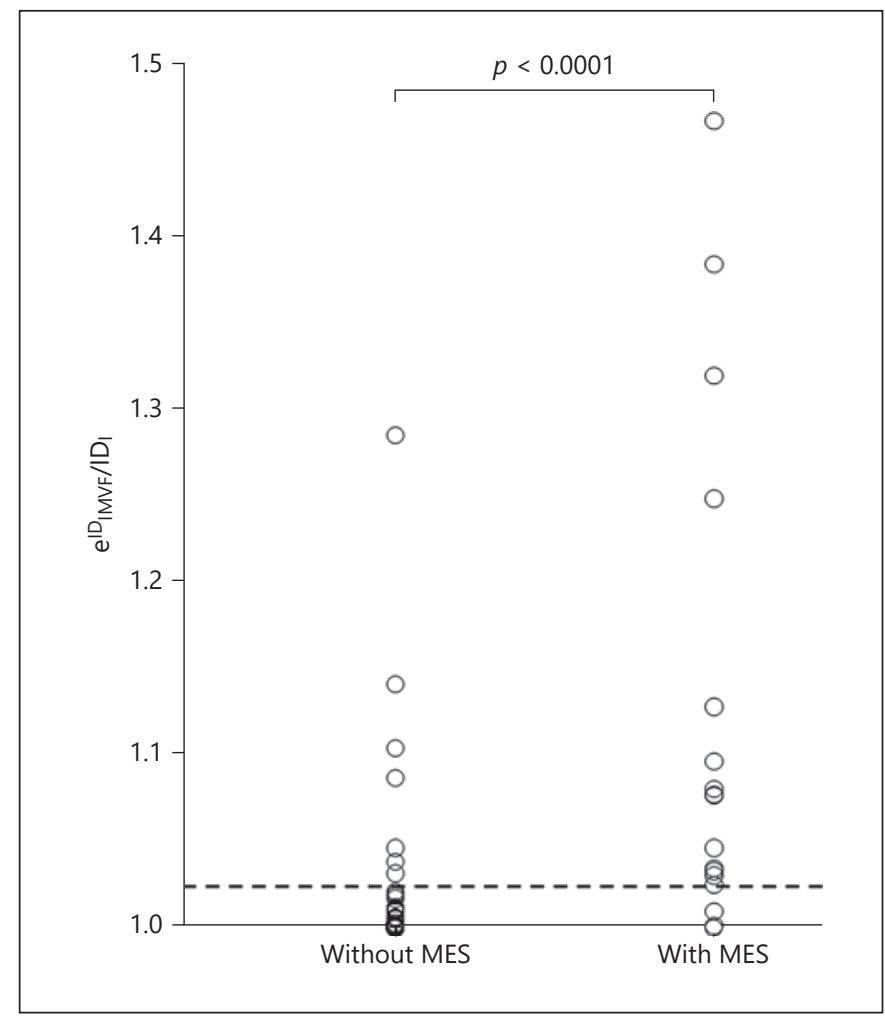

Fig. 3. Relationship between the $\mathrm{ID}_{\mathrm{IMVF}} / \mathrm{ID}_{1}$ ratio and development of MES during exposure of the carotid arteries. The vertical axis is shown as a natural exponential function of $\mathrm{ID}_{\mathrm{IMVF}} / \mathrm{ID}_{1}$. The dashed horizontal line $\left(\mathrm{e} 0.022=1.022\right.$ for $\left.\mathrm{ID}_{\mathrm{IMVF}} / \mathrm{ID}_{\mathrm{l}}\right)$ denotes the cutoff point lying closest to the upper left corner of the ROC curve for predicting the development of MES during exposure of the carotid arteries.

were classified as asymptomatic [20]. Thirty patients, including those 7 , had asymptomatic ICA stenosis. The mean $( \pm \mathrm{SD})$ degree of ICA stenosis overall was $88.1 \pm$ $9.3 \%$ (range $70-95$ ), and $\geq 70 \%$ stenosis or occlusion in the contralateral ICA was seen in 9 patients. All 70 patients visited our institute more than 14 days after the last ischemic attack and underwent CEA more than 30 days after the last ischemic attack because none had further ischemic events while awaiting surgery. Left-sided CEA was performed in 35 patients.

IMVF signals were identified in $46(66 \%)$ of the 70 patients studied; 1, 3, 4, and 38 patients had 4, 3, 2, and 1 IMVF signal, respectively. The interobserver (weighted $\kappa$ $=0.792$ ) agreement in the number of IMVF signals was excellent [21]. MES were detected in 17 (24\%) of the 70 patients when the ICA was manipulated to allow exposure from the carotid sheath. The number of MES ranged from 1 to 25 (mean: 7). The incidence of identification of
IMVF signals was significantly greater in patients with MES (94\%) than in those without (57\%; $p=0.0067)$. Figure 3 shows the relationship between $\mathrm{ID}_{\mathrm{IMVF}} / \mathrm{ID}_{1}$ and the development of MES. The $\mathrm{ID}_{\mathrm{IMVF}} / \mathrm{ID}_{1}$ ratio was significantly greater in patients with MES $(0.108 \pm 0.120)$ than in those without $(0.017 \pm 0.042 ; p<0.0001)$. The AUC for $\mathrm{ID}_{\mathrm{IMVF}} / \mathrm{ID}_{1}$ for prediction of the development of MES was 0.850 .

Of the 17 patients with MES, 8 (47.1\%) developed new postoperative ischemic lesions on DWI. None of the 53 patients without MES had such lesions. The $\mathrm{ID}_{\mathrm{IMVF}} / \mathrm{ID}_{1}$ ratio was significantly greater in patients with new postoperative ischemic lesions $(0.164 \pm 0.144)$ than in those without $(0.023 \pm 0.048 ; p=0.0018)$.

The sensitivity, specificity, and positive and negative predictive values for the identification of IMVF signals and $\mathrm{ID}_{\mathrm{IMVF}} / \mathrm{ID}_{1}$ are shown in Table 1 . In the $\mathrm{ID}_{\mathrm{IMVF}} / \mathrm{ID}_{1}$ ratio, the cutoff point lying closest to the upper left corner of the ROC curve for prediction of the development of MES was determined. The specificity and positive predictive value were significantly higher for $\mathrm{ID}_{\mathrm{IMVF}} / \mathrm{ID}_{1}$ than for identification of IMVF signals; however, no differences were seen in the sensitivity or negative predictive value.

Table 2 shows the results of the univariate analysis of factors related to the development of MES, except for $\mathrm{ID}_{\mathrm{IMVF}} / \mathrm{ID}_{\mathrm{l}}$. No significant associations were seen between any variables and the development of MES. Only $\mathrm{ID}_{\mathrm{IMVF}} / \mathrm{ID}_{1}$ was significantly associated with the development of MES (95\% CI 101.1-3,628.9; $p=0.0048$ ) on logistic regression analysis.

\section{Discussion}

The present study demonstrated that preoperative cervical carotid artery SMI ultrasound predicts the development of MES on TCD during exposure of the carotid arteries in CEA.

Previous studies reported that IMVF signals near the surface of the carotid plaque were seen in $45-65 \%$ of patients with carotid stenosis $[15,18]$; our data corresponded with this prevalence. One major finding of the present study was that the incidence of identification of IMVF signals was significantly greater in patients with MES than in those without. However, whereas the sensitivity and negative predictive value for the identification of IMVF signals for prediction of the development of MES were high $(>90 \%)$, the specificity and positive predictive value were low $(<50 \%)$. Thus, whereas the absence of IMVF signals suggests no occurrence of MES, the pres- 
Table 1. Sensitivity, specificity, and positive and negative predictive values for identification of IMVF signals and the $\mathrm{ID}_{\mathrm{IMVF}} / \mathrm{ID}_{1}$ ratio for prediction of the development of MES during exposure of the carotid arteries

\begin{tabular}{llrl}
\hline & $\begin{array}{l}\text { Identification of } \\
\text { IMVF signals }\end{array}$ & $\mathrm{ID}_{\mathrm{IMVF}} / \mathrm{ID}_{1}$ & $\begin{array}{l}\text { Statistical significance by } \\
\text { comparison of 95\% CI }\end{array}$ \\
\hline Sensitivity & $94(83-100)$ & $82(64-100)$ & No \\
Specificity & $43(30-57)$ & $87(78-96)$ & Yes \\
Positive predictive value & $35(21-48)$ & $67(48-87)$ & Yes \\
Negative predictive value & $96(88-100)$ & $94(87-100)$ & No \\
Cutoff point & & 0.022 & \\
\hline
\end{tabular}

Values are presented as percents (95\% CI) unless otherwise stated.

Table 2. Univariate analysis of factors related to the development of MES during exposure of the carotid arteries

\begin{tabular}{lcrc}
\hline \multirow{2}{*}{ Variables } & \multicolumn{2}{l}{ Development of MES } & \multirow{2}{*}{$p$ value } \\
\cline { 2 - 3 } & yes $(n=17)$ & no $(n=53)$ & \\
\hline Age, years & $71.2 \pm 8.4$ & $70.8 \pm 5.5$ & 0.4628 \\
Male sex & $14(82)$ & $49(92)$ & 0.3489 \\
Hypertension & $14(82)$ & $47(89)$ & 0.6777 \\
Diabetes mellitus & $4(24)$ & $17(32)$ & 0.5610 \\
Dyslipidemia & $15(88)$ & $47(89)$ & 0.9999 \\
Symptomatic lesion & $15(88)$ & $42(79)$ & 0.4984 \\
Degree of ICA stenosis, $\%$ & $87.6 \pm 9.5$ & $88.2 \pm 9.4$ & 0.7398 \\
Bilateral lesions & $1(6)$ & $8(15)$ & 0.4376 \\
Left-sided CEA & $11(65)$ & $24(45)$ & 0.2647 \\
\hline
\end{tabular}

Values are presented as means \pm SD or numbers (\%).

ence of IMVF signals does not always suggest the occurrence of MES.

The degree of change of IMVF signals synchronizing with heartbeat cycles implies the strength of the pulsatile flow of neovessels within the carotid plaque. This strength might be proportional to the volume of neovessels per unit volume of plaque, which is associated with plaque vulnerability, and is also influenced by the pulsatile flow of the carotid arteries themselves. Therefore, we normalized the change in IMVF signals using changes in the carotid artery lumen signals. As a result, a greater change in normalized IMVF signals synchronizing with heartbeat cycles was an independent predictor of the development of MES, and the quantitative assessment of such changes increased the specificity and positive predictive value for prediction of the development of MES. Another previous study reported that contrast-enhanced ultrasound using intravascular tracers containing less soluble gases detected neovessels in the carotid plaques with accuracy com- parable to that of SMI ultrasound [15], and its preoperative findings were found to be associated with the development of MES during CEA [12]. The predictive accuracy obtained in the present study for the development of MES during CEA for SMI ultrasound was equal to that for contrast-enhanced ultrasound [12]. However, patients with a previous allergic reaction to eggs cannot receive a current-generation contrast agent as an intravascular tracer for contrast-enhanced ultrasound because the lipid-stabilized suspension of the contrast agent contains egg yolk [21, 22]. These findings suggest that SMI ultrasound is a viable alternative to contrast-enhanced ultrasound for prediction of embolism during exposure of the carotid arteries in CEA.

The findings of this study can be applied to clamping of the common and external carotid arteries prior to manipulation of the carotid bulb and the ICA during exposure of the carotid artery in CEA [23]. This early clamping of the common and external carotid arteries has been 
shown to reverse the pressure direction (from the intracranial to the extracranial artery) in the ipsilateral ICA and prevent cerebral embolism during subsequent exposure of the carotid bulb and ICA [23]; however, it is also known to cause a longer duration of cerebral ischemia [23]. Given the present findings and data, the following practical clinical algorithm is proposed to prevent the development of MES in CEA. First, patients undergo preoperative cervical carotid artery SMI ultrasound. The $\mathrm{ID}_{\mathrm{IMVF}} / \mathrm{ID}_{1}$ ratio is calculated when the IMVF signals are visually identified. When the $\mathrm{ID}_{\mathrm{IMVF}} / \mathrm{ID}_{1}$ ratio is high $(>0.022)$, early clamping of the carotid arteries is carried out during carotid artery exposure. In contrast, this procedure is unnecessary when no IMVF signals are visually identified or when the $\mathrm{ID}_{\mathrm{IMVF}} / \mathrm{ID}_{1}$ ratio is low $(\leq 0.022)$. Based on this practical clinical algorithm, early clamping of the carotid arteries is recommended in only about $30 \%$ of our patients.

In general, $20-30 \%$ of patients do not show reliable results on TCD monitoring because of a failure to obtain an adequate bone window. Actually, 14\% of our patients showed such TCD monitoring findings. In addition, as few as 1-3 MES identified on TCD monitoring during exposure of the carotid arteries in CEA result in new postoperative ischemic lesions on magnetic resonance imaging $[24,25]$. MES tend to develop when the carotid bulb and ICA are manipulated $[24,25]$. Therefore, reversal of the pressure direction by clamping of the common and external carotid arteries should be done prior to the development of MES by such manipulation. These 2 findings explain the reason why preoperative cervical carotid artery SMI ultrasound rather than detection of MES on TCD should be used as a practical clinical algorithm for early clamping of the carotid arteries.

However, this study does have some limitations. First, observers visually distinguished the IMVF signals from other static enhancements such as intraplaque calcification. Although the interobserver agreement for identification of IMVF signals was excellent, the development of a method that automatically and accurately identifies IMVF signals would enable our findings to be more robust. Second, patients with chronic atrial fibrillation were excluded from the present study. Time-intensity curves of the IMVF signals were segmented into each heartbeat cycle based on gated electrocardiogram findings. This segmentation cannot be correctly performed in patients with chronic atrial fibrillation. Lastly, all patients studied underwent CEA more than 30 days after the last ischemic attack. According to an analysis of pooled data from randomized controlled trials of endarterectomy for symp- tomatic carotid stenosis, the shorter the period from the onset of the ischemic attack, the higher the incident of stroke recurrence; CEA should be performed within 2 weeks of the last ischemic attack [1]. Therefore, it remains unclear whether our findings are applicable to patients who undergo CEA within 2 weeks of the last ischemic attack.

\section{Conclusion}

Preoperative cervical carotid artery SMI ultrasound can predict the development of MES on TCD during exposure of the carotid arteries in CEA, and it may therefore be applicable as a preoperative tool to determine whether the carotid arteries should be clamped prior to manipulation of the carotid bulb and ICA.

\section{Statement of Ethics}

This prospective observational study was carried out in accordance with the guidelines of the World Medical Association and the Declaration of Helsinki, and the protocol was reviewed and approved by the Iwate Medical University School of Medicine Institutional Ethics Committee (No. MH2019-061). Written informed consent was obtained from all of the patients or their next of kin before this study began.

\section{Conflict of Interest Statement}

The author (Kuniaki Ogasawara) declares the following potential conflict of interests with respect to the research, authorship, and/or publication of this article: consigned research funds from Nihon Medi-Physics Co., Ltd. (JPY 3,000,000).

\section{Funding Sources}

This work was partly supported by Grants-in-Aid for Scientific Research from the Japan Society for the Promotion of Science (No. 18K09002 and 20K16556).

\section{Author Contributions}

T. Chiba and K. Ogasawara: study conception and design; acquisition, analysis, and interpretation of data; drafting of this article; critical revision of this work for important intellectual content; final approval of the version to be published; and agreement to being accountable for all aspects of the work in ensuring that questions related to the accuracy or integrity of any part of this work are appropriately investigated and resolved. S. Fujiwara, K. 
Oura, K. Yoshida, Y. Kubo, and T. Maeda: study conception and design, critical revision of this article for important intellectual content, final approval of the version to be published, and agreement to being accountable for all aspects of the work in ensuring that questions related to the accuracy or integrity of any part of this work are appropriately investigated and resolved. K. Oikawa, K.
Chida, M. Kobayashi, and R. Itabashi: acquisition of data, critical revision of this article for important intellectual content, final approval of the version to be published, and agreement to being accountable for all aspects of the work in ensuring that questions related to the accuracy or integrity of any part of the work are appropriately investigated and resolved.

\section{References}

1 Rothwell PM, Eliasziw M, Gutnikov SA, Fox AJ, Taylor DW, Mayberg MR, et al.; Carotid Endarterectomy Trialists' Collaboration. Analysis of pooled data from the randomised controlled trials of endarterectomy for symptomatic carotid stenosis. Lancet. 2003 Jan;361(9352):107-16.

2 Walker MD. Executive Committee for the Asymptomatic Carotid Atherosclerosis Study. Endarterectomy for asymptomatic carotid artery stenosis. JAMA. 1995;273(18):1421-8.

3 Barnett HJ, Taylor DW, Haynes RB, Sackett DL, Peerless SJ, Ferguson GG, et al.; North American Symptomatic Carotid Endarterectomy Trial Collaborators. Beneficial effect of carotid endarterectomy in symptomatic patients with high-grade carotid stenosis. N Engl J Med. 1991 Aug;325(7):445-53.

4 Spencer MP. Transcranial Doppler monitoring and causes of stroke from carotid endarterectomy. Stroke. 1997 Apr;28(4):685-91.

5 Wolf O, Heider P, Heinz M, Poppert H, Sander D, Greil O, et al. Microembolic signals detected by transcranial Doppler sonography during carotid endarterectomy and correlation with serial diffusion-weighted imaging. Stroke. 2004 Nov;35(11):e373-5.

6 Verhoeven BA, de Vries JP, Pasterkamp G, Ackerstaff RG, Schoneveld AH, Velema E, et al. Carotid atherosclerotic plaque characteristics are associated with microembolization during carotid endarterectomy and procedural outcome. Stroke. 2005 Aug;36(8):173540.

7 Rosario JA, Hachinski VC, Lee DH, Fox AJ. Adverse reactions to duplex scanning. Lancet. 1987 Oct;2(8566):1023.

8 van Dijk AC, Truijman MT, Hussain B, Zadi T, Saiedie G, de Rotte AA, et al. Intraplaque hemorrhage and the plaque surface in carotid atherosclerosis: the plaque at risk study (PARISK). AJNR Am J Neuroradiol. 2015 Nov;36(11):2127-33.

9 Jander S, Sitzer M, Schumann R, Schroeter M, Siebler M, Steinmetz H, et al. Inflammation in high-grade carotid stenosis: a possible role for macrophages and $\mathrm{T}$ cells in plaque destabilization. Stroke. 1998 Aug;29(8):1625-30.
10 Staub D, Partovi S, Schinkel AF, Coll B, Uthoff $\mathrm{H}$, Aschwanden $\mathrm{M}$, et al. Correlation of carotid artery atherosclerotic lesion echogenicity and severity at standard US with intraplaque neovascularization detected at contrast-enhanced US. Radiology. 2011 Feb; 258(2):618-26.

11 Shimada Y, Oikawa K, Fujiwara S, Ogasawara Y, Sato Y, Narumi S, et al. Comparison of Three-Dimensional T1-Weighted Magnetic Resonance and Contrast-Enhanced Ultrasound Plaque Images for Severe Stenosis of the Cervical Carotid Artery. J Stroke Cerebrovasc Dis. 2017 Sep;26(9):1916-22.

12 Oikawa K, Kato T, Oura K, Narumi S, Sasaki M, Fujiwara S, et al. Preoperative cervical carotid artery contrast-enhanced ultrasound findings are associated with development of microembolic signals on transcranial Doppler during carotid exposure in endarterectomy. Atherosclerosis. 2017 May;260:87-93.

13 Sztajzel R, Momjian S, Momjian-Mayor I, Murith N, Djebaili K, Boissard G, et al. Stratified gray-scale median analysis and color mapping of the carotid plaque: correlation with endarterectomy specimen histology of 28 patients. Stroke. 2005 Apr;36(4):741-5.

14 Gaunt ME, Brown L, Hartshorne T, Bell PR, Naylor AR. Unstable carotid plaques: preoperative identification and association with intraoperative embolisation detected by transcranial Doppler. Eur J Vasc Endovasc Surg. 1996 Jan;11(1):78-82.

15 Zamani M, Skagen K, Scott H, Lindberg B, Russell D, Skjelland M. Carotid Plaque Neovascularization Detected With Superb Microvascular Imaging Ultrasound Without Using Contrast Media. Stroke. 2019 Nov;50(11): 3121-7.

16 Zhou Y, Wang C. Superb microvascular imaging for detecting neovascularization of carotid plaque compared with contrast-enhanced ultrasound: A protocol for systematic review and meta analysis. Medicine (Baltimore). 2020 Aug;99(35):e21907.

17 Forsberg F, Machado P, Stanczak M, Farber J, DiMuzio P, Needleman L. Assessing carotid plaque neovascularity and calcifications in patients prior to endarterectomy. J Vasc Surg. 2019 Oct;70(4):1137-44.
18 Chen X, Wang H, Jiang Y, Li J, Li N, Kong J, et al. Neovascularization in carotid atherosclerotic plaques can be effectively evaluated by superb microvascular imaging (SMI): initial experience. Vasc Med. 2020 Aug;25(4): 328-33.

19 Consensus Committee of the Ninth International Cerebral Hemodynamic Symposium. Basic identification criteria of Doppler microembolic signals. Stroke. 1995 Jun;26(6):1123.

20 Brott TG, Hobson RW 2nd, Howard G, Roubin GS, Clark WM, Brooks W, et al.; CREST Investigators. Stenting versus endarterectomy for treatment of carotid-artery stenosis. N Engl J Med. 2010 Jul;363(1):11-23.

21 Saito K, Nagatsuka K, Ishibashi-Ueda H, Watanabe A, Kannki H, Iihara K. Contrast-enhanced ultrasound for the evaluation of neovascularization in atherosclerotic carotid artery plaques. Stroke. 2014 Oct;45(10):3073-5.

22 Varetto G, Gibello L, Castagno C, Quaglino S, Ripepi M, Benintende E, et al. Use of contrastenhanced ultrasound in carotid atherosclerotic disease: limits and perspectives. BioMed Res Int. 2015;2015:293163.

23 Kobayashi M, Ogasawara K, Inoue T, Saito H, Komoribayashi N, Suga Y, et al. Urgent endarterectomy using pretreatment with free radical scavenger, edaravone, and early clamping of the parent arteries for cervical carotid artery stenosis with crescendo transient ischemic attacks caused by mobile thrombus and hemodynamic cerebral ischemia. Case report. Neurol Med Chir (Tokyo). 2007 Mar;47(3): 121-5.

24 Ogasawara K, Suga Y, Sasaki M, Chida K, Kobayashi $\mathrm{M}$, Yoshida $\mathrm{K}$, et al. Intraoperative microemboli and low middle cerebral artery blood flow velocity are additive in predicting development of cerebral ischemic events after carotid endarterectomy. Stroke. 2008 Nov; 39(11):3088-91.

25 Aso K, Ogasawara K, Sasaki M, Kobayashi M, Suga Y, Chida K, et al. Preoperative cerebrovascular reactivity to acetazolamide measured by brain perfusion SPECT predicts development of cerebral ischemic lesions caused by microemboli during carotid endarterectomy. Eur J Nucl Med Mol Imaging. 2009 Feb;36(2): 294-301. 